\title{
Two cases of opioid rotation applied to patients with chronic pain
}

\author{
Gun Woo Kim, Sung An Kang, Young Deog Cha, Doo Ik Lee, and Jang Ho Song \\ Department of Anesthesiology and Pain Medicine, Inha University, Inha University Hospital, Incheon, Korea
}

Opioid rotation, defined as a change in the opioid drug or route of administration to optimize the therapeutic effects, can be applied to both cancer pain and chronic non-cancer pain [1]. Although it is unclear why opioid rotation improves the analgesic effect, it is believed that the $\mu$-opioid receptor affects patients differently and that the effect of opioid rotation is related to the incomplete cross-tolerance of opioids [2]. Two successful cases of opioid rotation are reported herein.

In September 2007, a 46-year-old man felt a sudden pain at both upper extremities of the C5 dermatome. An intramedullary tumor was diagnosed by MRI of the C-spine. After surgery, the patient complained of severe pain to his arms. Although the patient was taking oxycodone CR $80 \mathrm{mg} /$ day prior to coming to our clinic in March 2010, his pain was not controlled and had a visual analogue scale (VAS) score of $8 / 10$. Therefore, opioid rotation was performed from oxycodone CR to morphine. Instead of oxycodone CR $80 \mathrm{mg} /$ day, the patient was prescribed morphine $90 \mathrm{mg} /$ day, a $25 \%$ reduction of the calculated equianalgesic dose, and a rescue dose of hydromorphone IR $8 \mathrm{mg} /$ day. On the second visit, the patient had an unsatisfactory VAS score of $7 / 10$, and the morphine dosage was increased to $120 \mathrm{mg} /$ day. On the third visit, the patient's VAS score decreased to 5/10. Two follow-up visits showed VAS scores of $4 / 10$ and 5/10, with the pain being well regulate. On a subsequent visit, however, the patient began to complain of severe constipation. Opioid rotation was commenced again, and the patient was prescribed an equianalgesic dose of hydromorphone SR, $32 \mathrm{mg} /$ day, instead of morphine $120 \mathrm{mg} /$ day. After 7 days, the VAS score showed a satisfactory controlled pain level of $4 / 10$, and the constipation also improved. During 6 months of follow-up visits, the patient's VAS score remained at a manageable level of between $4 / 10$ and $6 / 10$ under the same dosage, and there were no particular adverse effects.

The second case involved a 34-year-old man with Marfan's syndrome who received two aortic graft operations and one Bentall operation for chronic aortic dissection from 1995 to 2009. During the final surgery in 2009, the patient developed spinal cord ischemia causing paralysis to the lower extremities. After the surgery, due to chronic severe pain in both lower extremities, the patient received continuous opioid administration. Before being referred to our hospital, the patient was administered oxycodone CR $160 \mathrm{mg} /$ day and was receiving treatment for mental status changes and pinpoint pupils due to the high opioid dosage. In September 2012, the patient was seen at our hospital. Although his VAS score was 10/10, the repeated mental status changes made it extremely difficult to increase the opioid dosage. Therefore, opioid rotation was performed from oxycodone CR to morphine. Instead of oxycodone CR $160 \mathrm{mg} /$ day, the patient was administered morphine $180 \mathrm{mg} /$ day, a $25 \%$ reduction of the calculated equianalgesic dose, and a rescue dose of hydromorphone IR $8 \mathrm{mg} /$ day. During the second visit one week later, the patient still felt severe pain, with a VAS score of $8 / 10$. The patient was directed to increase the dosage every two days until there was an improvement in pain but to discontinue the increase in the case of adverse side effects. On the third visit, the patient was taking morphine $480 \mathrm{mg} /$ day with an improved VAS pain level of $4 / 10$. There were no adverse side effects at this dosage. To date, the same dosage has not resulted in any adverse

Corresponding author: Young Deog Cha, M.D., Department of Anesthesiology and Pain Medicine, Inha University, Inha University Hospital, Sinheung-dong 3-ga, Jung-gu, Incheon 400-711, Korea. Tel: 82-32-890-3968, Fax: 82-32-881-2476, E-mail: ydchaan@inha.ac.kr

(c) This is an open-access article distributed under the terms of the Creative Commons Attribution Non-Commercial License (http:// creativecommons.org/licenses/by-nc/3.0/), which permits unrestricted non-commercial use, distribution, and reproduction in any medium, provided the original work is properly cited. 
effects in this patient.

In the first case, although the patient was administered oxycodone CR $80 \mathrm{mg} /$ day, the pain was not controlled and had a VAS level of $8 / 10$. Although the pain was managed after rotation to morphine, further opioid rotation was required due to severe constipation. In the second case, the patient had a VAS score of 10/10 despite receiving oxycodone CR $160 \mathrm{mg}$ /day and required an increase in the dosage, but due to adverse effects, opioid rotation was performed. Indications for opioid rotation are (1) the occurrence of severe adverse effects, (2) poor analgesic efficacy despite an increase in dosage, (3) a problematic drug-drug interaction, (4) a change in clinical status or clinical setting, and (5) financial considerations [3].

The conversion ratio should be determined by considering the unique clinical situation of patient [4]. Generally, a two-step process is involved in opioid rotation. The first step is to automatically reduce the equianalgesic dose by $25-50 \%$. Variation among individual patients in opioid rotation and the occurrence of incomplete cross-tolerance during chronic treatment may cause the physician to underestimate the potency of the new opioid. However, the automatic dose reduction does not apply to methadone and transdermal fentanyl patches. When calculating the reduction, one must use clinical judgment, tak- ing into account age, associated diseases, and race. The second step involves additional assessment of the patient's pain and consideration of medical or psychosocial factors. During this step, the dose can be further adjusted by an additional 15-30\%. For example, if the pain is severe, one should not apply the automatic reduction in the first step, and if the pain is not severe and there are side effects, an additional reduction may be required. For breakthrough pain, a short-acting supplemental dose of only $5-15 \%$ of the daily opioid dose, referred to as a rescue dose, should be calculated and administered appropriately [3].

In the first case, in which the patient received an equianalgesic dose from oxycodone, the second opioid rotation dose was effective because it did not produce any side effects and controlled the pain well. In the second case, the dosage of morphine increased twice, and it was not possible to achieve dose titration with oxycodone due to adverse effects. Presently, this patient's pain control is well managed and without any side effects, which is a meaningful outcome.

In conclusion, if an increase in opioid dosage is difficult in chronic pain patients because the pain is not controlled or due to adverse side effects, opioid rotation may improve pain management.

\section{References}

1. Quigley C. Opioid switching to improve pain relief and drug tolerability. Cochrane Database Syst Rev 2004; (3): CD004847.

2. Pasternak GW. Incomplete cross tolerance and multiple mu opioid peptide receptors. Trends Pharmacol Sci 2001; 22: 67-70.

3. Fine PG, Portenoy RK. Establishing "best practices" for opioid rotation: conclusions of an expert panel. J Pain Symptom Manage 2009; 38: 418-25.

4. Knotkova H, Fine PG, Portenoy RK. Opioid rotation: the science and the limitations of the equianalgesic dose table. J Pain Symptom Manage 2009; 38: 426-39. 